\title{
Obstructive right paraduodenal hernia: A case report
}

\author{
Abdalla R.o., MBBS and Mungatana C., MBChB, MCS (ECSA), Department of Surgery, Rift Valley \\ Provincial General Hospital, Nakuru, Kenya \\ Corresponding author: Dr. Abdalla R.O., Department of Surgery, Rift Valley Provincial General Hospital, \\ Nakuru, Kenya. P.O. Box 71, Nakuru, Email: yrama7@yahoo.com
}

\section{Summary}

Intestinal obstruction is a prevalent condition in rural Kenya. Common aetiologies include sigmoid volvuli, strangulated hernias, intussusception and in children, congenital gut malformations. Paraduodenal hernia causing intestinal obstruction has not been encountered in our practice.

\section{Case}

A twelve year old patient presented with a one day history of abdominal distention, worsening colicky abdominal pain associated with two bouts of bilious emesis.

Examination revealed a sick-looking patient with a pulse rate of $98 / \mathrm{min}$, respiratory rate of $22 / \mathrm{min}$, blood pressure of $110 / 70$ and temperature of $36.9^{\circ} \mathrm{C}$. There was no pallor, jaundice or features of dehydration noted.

There was notable upper abdominal distension with significant tenderness. The rest of the abdomen was unremarkable. Bowel sounds were normal and a rectal examination revealed an empty rectum.

A plain erect abdominal $\mathrm{X}$-ray revealed distended loops of small bowel, mainly on the right aspect of the abdomen and multiple air-fluid levels. An impression of acute small bowel obstruction was made and an urgent laparotomy planned.

Intra-operatively, initial finding was that of loops of small intestines obstructed in a clear sac that was on the right side of the abdomen, deep to the gastrocolic ligament (Figure 1).
The point of herniation was deep to the posterior leaf of the transverse mesocolon, and was not in communication with the lesser sac. Also noted was a mobile splenic flexure that was adhered to the caecum (Figure 2).

The loops of small intestines were manually reduced through the point of herniation (Figure 3) and after successful manipulation the hernial orifice was defined as shown in Figure 4. The sac was left intact. The small intestines were slightly oedematous but viable.

Figure 1: The loops of bowel were trapped in sac on the right side of the abdomen

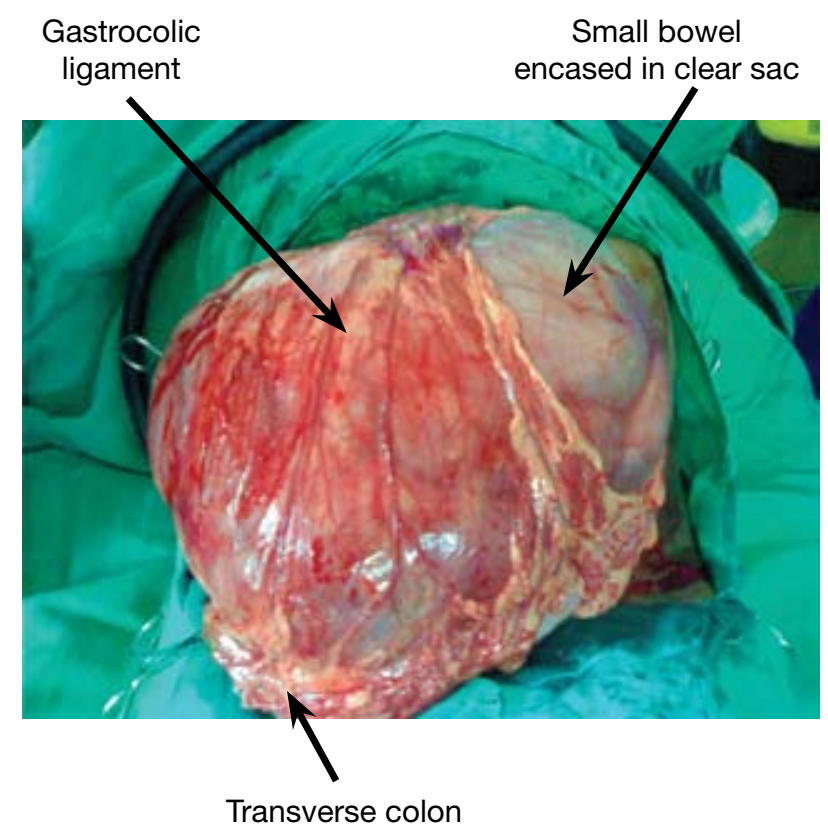


Figure 2: Splenic flexure adhered to caecum

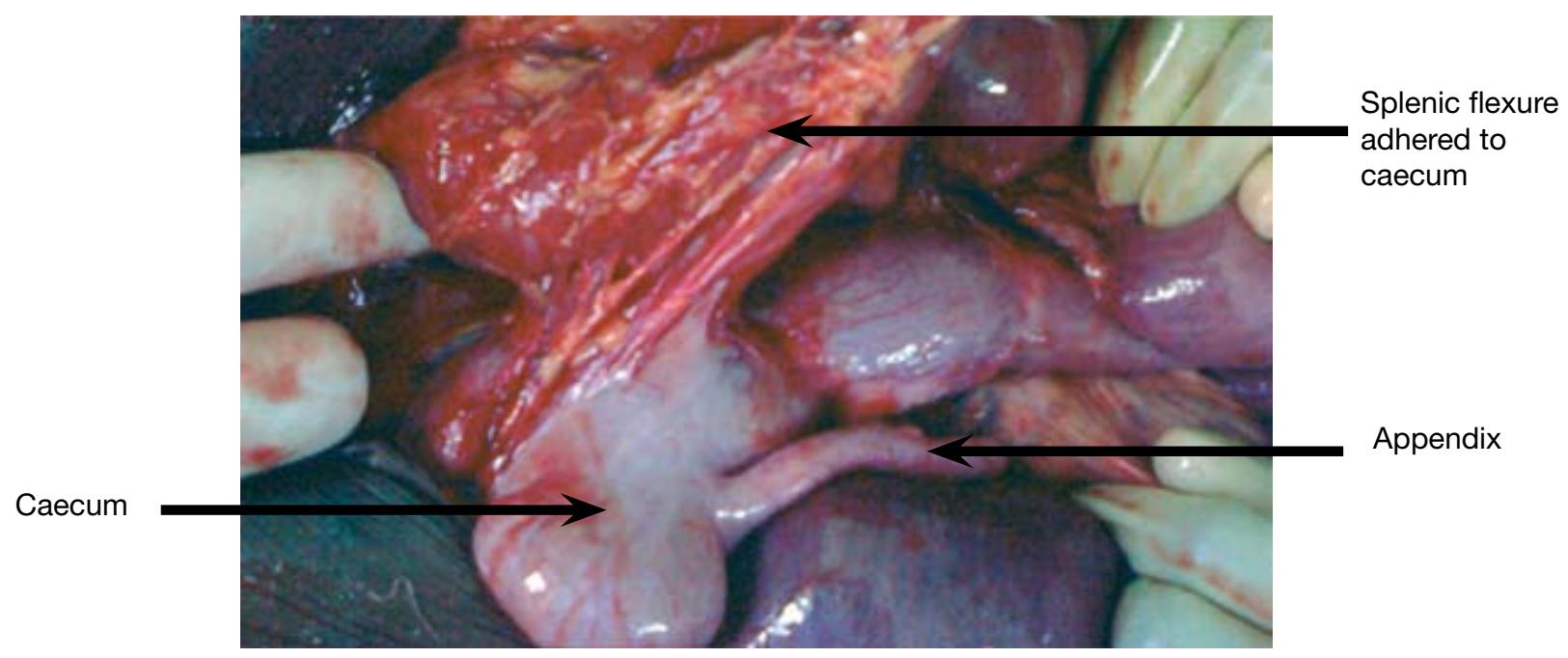

Figure 3: Manual reduction of herniated gut

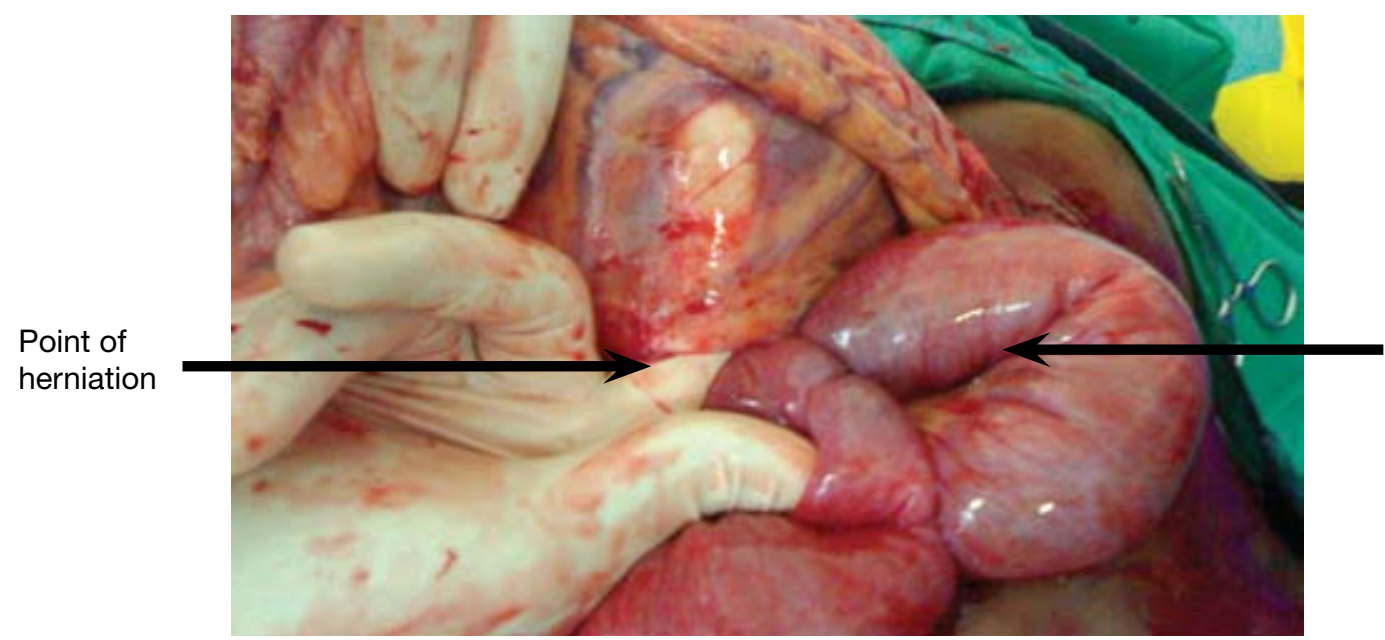

Loops of gut manipulated from point of herniation

\section{Figure 4: Point of herniation}

Point of herniation after manipulation of small bowel

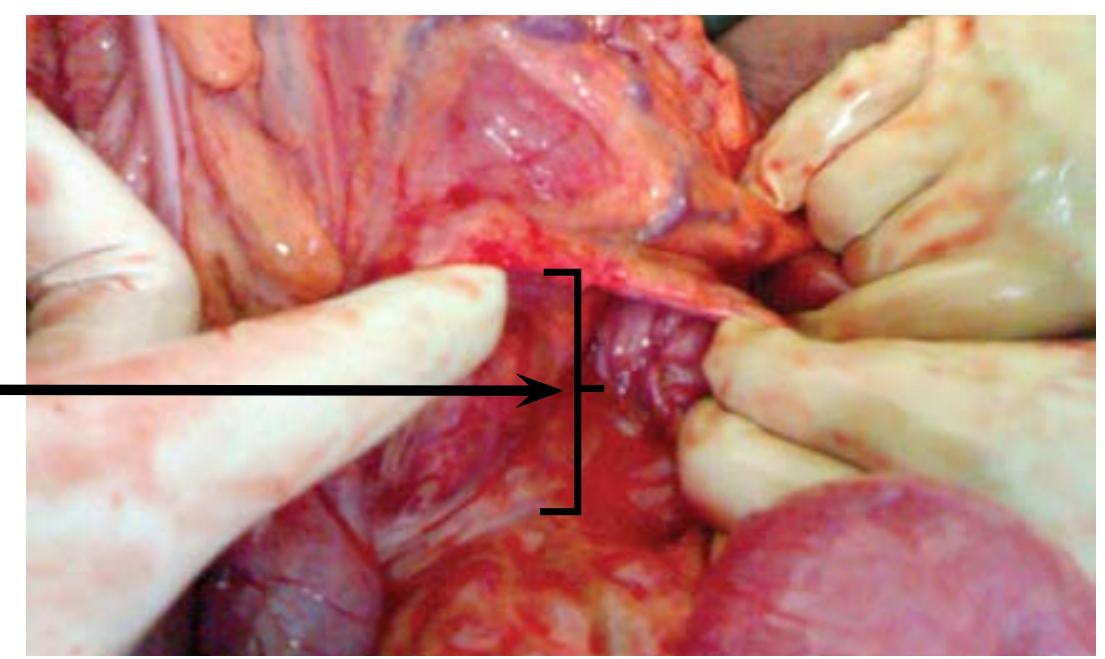


The hernial orifice was closed by suture and the patient made an uneventful recovery and was discharged five days later. On follow up the patient has remained well.

\section{Discussion}

Para duodenal hernia was initially described by Klob in 1861. The hernias are divided into right and left with the left three times more common (9). The pathogenesis of paraduodenal hernia is uncertain. They were previously thought to be acquired herniations of small bowel into one of the paraduodenal fossae, but it is now generally accepted that they are congenital in origin and caused by abnormal mid-gut rotation causing the small intestines to be entrapped behind the right mesocolon fusing to the retroperitoneum. Along with descent of the caecum, the small intestine is imprisoned (6).

These hernias are an important consideration in acute small bowel obstruction in the virgin abdomen. The pre-arterial segments of the mid-gut are in a non-rotated position in the right abdomen and afferent and efferent loops of small intestines enter and exit the neck of the sac. Rarely, the right colon can be contained within the sac. The orifice is to the right of the midline and is usually oriented slightly inferiorly (8). The anterior free edge of the neck of the sac contains the superior mesenteric artery and ileocolic arteries, which must be avoided during surgery for the obstruction.

These hernias carry a 50\% lifetime risk of strangulation $(6,9)$. Furthermore the retromesocolic location of the hernia may mask any features of peritonitis that may occur leading to poorer outcomes.

The diagnosis requires a high index of suspicion. The most common presentation is acute small bowel obstruction. Abdominal $x$-rays may show distended loops of small bowel accumulated on one side of the abdomen, but this does not usually diagnose the cause of the obstruction. In $70-80 \%$ of patients, there is a long standing history of recurrent subacute obstruction or vague abdominal pain. In these patients, bowel contrast radiography may show a portion of small bowel confined within an area of the abdomen despite changes in the patients position, aiding in diagnosis $(8,10)$.

The management of paraduodenal hernias is surgical with reduction of the loops of small bowel if possible or incision of the inferior edge of the sac to release the gut, define the hernia orifice and suturing (2).

Diagnosis is often made during surgery and early intervention is recommended to avoid any complications.

\section{Acknowledgements}

I would like to thank Dr K.C. Lakati and Dr S.K. Kabinga for their support during the preparation of this paper, and Mr P. Oduor, Head of Department of Surgery, Provincial General Hospital, Nakuru, for his support, constructive criticism and the institution for its assistance.

\section{References}

1. Andrews E. Duodenal hernia, a misnomer. Surg. Gynecol. Obstet. 1923; 37: 740-750.

2. Isabel L., Birell S. and Patkin M. Paraduodenal hernia. Aust. N. Z. J. Surg. 1995; 65: 64-66.

3. Berardi R.S. Paraduodenal hernia. Surg. Gynecol. Obstet. 1981; 152: 99-110.

4. Dott N.M. Anomalies of intestinal rotation; their embryological and surgical aspect; Brit. J. Surg. 1923; 11: 251-285.

5. Collander C.A., Rusk G.Y. and Nemir A. Mechanisms, symptoms and treatment of hernia into the descending mesocolon; left duodenal hernia. Surg. Gynecol.Obstet. 1935; 60: 1052-1071.

6. Raymond S., Tong K., Sengupta S., et al. Left paraduodenal hernia. Case report. ANZ J. Surg. 2002; 72: 69-71.

7. Bartlett M.K., Wang C. and Williams W.H. The surgical management of paraduodenal hernia. Ann. Surg. 1968; 168: 249-254.

8. Surgery: Scientific principles and practice - Lipincott and Raven: Second edition, Lazar J. Greenfield. 1997.

9. Sinohara T., Okugawa K. and Furuta C. Volvulus of the small intestine caused by right paraduodenal hernia. J. Pediat. Surg. 2004; 39(2)(suppl)3): E15.

10. Lee T.K.Y., Voon F.C.T., Chow K.W., et al. Unusual variant of right paraduodenal hernia. Case report. ANZ J. Surg. 1990; 60: 483-485. 